\title{
Ontology-Based Fuzzy-Syllogistic Reasoning
}

\author{
$\underline{\text { Mikhail Zarechnev }}^{(\bowtie)}$ and Bora İ Kumova \\ Department of Computer Engineering, İzmir Institute of Technology, \\ 35430, Urla/İzmir, Turkey \\ \{mikhailzarechnev, borakumova\} @iyte.edu.tr
}

\begin{abstract}
We discuss the Fuzzy-Syllogistic System (FSS) that consists of the well-known 256 categorical syllogisms, namely syllogistic moods, and FuzzySyllogistic Reasoning (FSR), which is an implementation of the FSS as one complex approximate reasoning mechanism, in which the 256 moods are interpreted as fuzzy inferences. Here we introduce a sample application of FSR as ontology reasoner. The reasoner can associate up to 256 possible fuzzyinferences with truth ratios in [0,1] for every triple concept relationship of the ontology. We further discuss a transformation technique, by which the truth ratio of a fuzzy-inference can increase, by adapting the fuzzy-quantifiers of a fuzzy-inference to the syllogistic logic of the sample propositions.
\end{abstract}

Keywords: Categorical syllogisms - Approximate reasoning - Ontologies · Fuzzy-logic

\section{Introduction}

Reasoning is the ability to make inferences and automated reasoning is an area of computer science and mathematical logic, dedicated to understanding different aspects of reasoning and concerned with building computing systems that automate this process. Although the term can be applied to various reasoning tasks, usually automated reasoning is considered with different forms of valid deductive reasoning, like in various applications of automated theorem proving or formal verification [11].

Reasoning with intermediate quantifiers is called fuzzy syllogistic reasoning [16], where a syllogism is an inference rule that consists of deducing a new quantified statement from one or several quantified statements.

Based on ideas of syllogistic reasoning we proposed the Fuzzy-Syllogistic System (FSS) [6], which attempts to integrate both approaches, approximate and exact reasoning, in one system.

The work is organized as follows: in chapters 2 and 3 we discuss syllogisms and their applications, in particular the concepts of the fuzzy-syllogistic system. In chapter 4 we provide a short description of ontology. Finally in chapters 5 we present the idea of ontology-based syllogistic reasoning.

\section{Classical Syllogisms}

As an inference scheme, a syllogism may generally be expressed in the form: 


$$
\begin{aligned}
& \psi_{1} A \text { are } B \\
& \frac{\psi_{2} C \text { are } D}{\psi_{3} E \text { are } F}
\end{aligned}
$$

where $\psi_{1}, \psi_{2}$ and $\psi_{3}$ are numerical, or more general, fuzzy quantifiers (e.g. few, many, most ), and A, B, C, D, E and F are crisp or fuzzy predicates. The predicates A, B, ... $\mathrm{F}$ are assumed to be related in a specific way, giving rise to different types of syllogisms [16].

A categorical syllogism can be defined as a logical argument that is composed of two logical propositions for deducing a logical conclusion, where the propositions and the conclusion each consist of a quantified relationship between two objects [1], [14].

\subsection{Syllogistic Propositions}

A syllogistic proposition or synonymously categorical proposition specifies a quantified relationship between two objects. We shall denote such relationships with the operator $\psi$. Four different types are distinguished $\psi=\{\mathrm{A}, \mathrm{E}, \mathrm{I}, \mathrm{O}\}$ :

\begin{tabular}{|l|l|l|l|l|l|l|}
\hline A & Universal Affirmative & All S are P & & E & Universal Negative & All S are not P \\
\hline I & Particular Affirmative & Some S are P & & O & Particular Negative & Some S are not P \\
\hline
\end{tabular}

\subsection{Syllogistic Figures}

A syllogism consists of the three propositions: major premise, minor premise and conclusion. The first proposition consist of a quantified relationship between the objects $\mathrm{M}$ and $\mathrm{P}$, the second proposition of $\mathrm{S}$ and $\mathrm{M}$, the conclusion of $\mathrm{S}$ and $\mathrm{P}$ (Table 1).

Table 1. Syllogistic figures

\begin{tabular}{|l|c|c|c|c|}
\hline Figure Name & I & II & III & IV \\
\hline Major Premise & $\mathrm{M} \psi \mathrm{P}$ & $\mathrm{P} \psi \mathrm{M}$ & $\mathrm{M} \psi \mathrm{P}$ & $\mathrm{P} \psi \mathrm{M}$ \\
Minor Premise & $\mathrm{S} \psi \mathrm{M}$ & $\mathrm{S} \psi \mathrm{M}$ & $\mathrm{M} \psi \mathrm{S}$ & $\mathrm{M} \psi \mathrm{S}$ \\
Conclusion & $\mathrm{S} \psi \mathrm{P}$ & $\mathrm{S} \psi \mathrm{P}$ & $\mathrm{S} \psi \mathrm{P}$ & $\mathrm{S} \psi \mathrm{P}$ \\
\hline
\end{tabular}

Note the symmetrical combinations of the objects. Since the proposition operator may have four values for $\psi, 64$ syllogistic moods are possible for every figure and 256 moods for all four figures in total.

\section{The Fuzzy Syllogistic System}

The proposed fuzzy-syllogistic system (FSS) is a complex model for approximate reasoning. It may be used for constructing hybrid systems that can reason deductively over emergent data concepts and their relationships. Here we improve the mathematical model of syllogistic system, presented in [5], [6] and [7]. 


\subsection{Syllogistic System}

For three sets, there are seven possible subsets in a Venn diagram (Fig. 1). These subsets or spaces constitute the basic data of modelling the syllogistic system (Table 2).

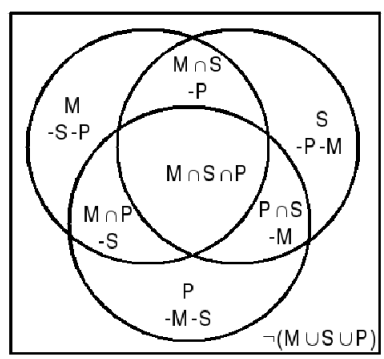

Fig. 1. Venn diagram for three sets

In an algorithmic implementation of the syllogistic system, we get 96 distinct space combinations, where everyone contains elements from all three sets simultaneously. We will refer to these combinations as the syllogistic cases.

Table 2. Identification of the seven possible subsets of three sets as distinct spaces

\begin{tabular}{|c|c|c|c|c|c|c|c|}
\hline Space ID & $\delta_{1}$ & $\delta_{2}$ & $\delta_{3}$ & $\delta_{4}$ & $\delta_{5}$ & $\delta_{6}$ & $\delta_{7}$ \\
\hline Subset & $\mathrm{S}-(\mathrm{P}+\mathrm{M})$ & $\mathrm{P}-(\mathrm{M}+\mathrm{S})$ & $\mathrm{M}-(\mathrm{S}+\mathrm{P})$ & $(\mathrm{M} \cap \mathrm{S})-\mathrm{P}$ & $(\mathrm{M} \cap \mathrm{P})-\mathrm{S}$ & $(\mathrm{S} \cap \mathrm{P})-\mathrm{M}$ & $\mathrm{M} \cap \mathrm{S} \cap \mathrm{P}$ \\
\hline
\end{tabular}

Every proposition of everyone of the 256 moods matches some of these 96 syllogistic cases. By relating the number of true and false matching cases per mood we calculate the degree of validity (or truth ratio) of that mood. More precisely, for a given mood, all 96 cases are checked against the premises and the conclusion and the number of true and false matching cases are identified. The ratio between matched true and false cases becomes the truth ratio $\tau=[0,1]$. Based on $\tau$, we can then judge about the accuracy of an inferred conclusion from given premises.

\subsection{Fuzzification}

Since the vast majority of syllogistic moods are invalid, we had to introduce the term of fuzzy-syllogistic reasoning (FSR) to generalize reasoning scheme and extend the possible number of valid syllogistic structures. According to the structure of syllogism we applied the fuzzification in two ways by using fuzzy quantifiers and defining fuzzy sets [8].

Let us consider mood AIA of figure 1:

\section{A: All $\mathrm{M}$ are $\mathrm{P} \quad$ I: Some $\mathrm{S}$ are $\mathrm{M}$ A: All $\mathrm{S}$ are $\mathrm{P}$}

According to the rules of classical logic this syllogism is not valid; there are only 6 valid moods for Figure 1 and AIA not in these list. For this mood we found 10 cases that satisfy premises, but only 4 of them satisfy conclusion and premises at the same 
time, so we can calculate true ratio $\tau=4 / 10$ (Fig. 2 a). For elements in $S$ that are not shared in $\mathrm{M}$ and $\mathrm{P}$, the conclusion becomes wrong (Fig. 2 b)).

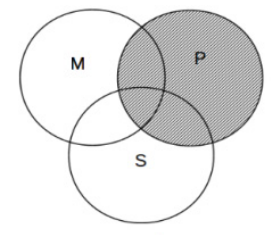

a)

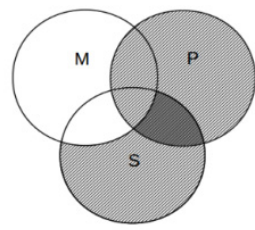

b)

Fig. 2. True a) and false b) syllogistic cases of the mood AIA1

Using fuzzy-quantification we can achieve fully true conclusion. Indeed if we replace the universal quantifier $A$ in the fuzzy-quantifier A': AlmostAll, the resulting syllogism became true:

\section{A: All $\mathrm{M}$ are $\mathrm{P} \quad$ I: Some $\mathrm{S}$ are $\mathrm{M} \quad \mathbf{A}^{\prime}$ : AlmostAll $\mathrm{S}$ are $\mathrm{P}$}

In certain situations, by taking some assumptions, we can replace one quantifier by another. More specifically, we can introduce another quantifier like A':AlmostAll or E':AlmostNone. Obviously, quantifier A' can be considered as a special case of quantifier I. Likewise, quantifier $\mathrm{E}^{\prime}$ is a special case of quantifier O:NotSome.

Returning to the given example, "AlmostAll $\mathrm{S}$ are $\mathrm{P}$ " means that the proportion of elements of $S$ being elements of $P$ is "very very important". In other words, the proportion in $\mathrm{S}$ not being $\mathrm{P}$ is "very very weak". Where the proportion of elements of set $S$ in the intersection with $P$ to cardinality of $S$ (refers to $A^{\prime}$ ) is close to 1 and proportion for I' is close to 0 .

Taking this into account we can replace quantifier A by I and $\mathrm{E}$ by $\mathrm{O}$ respectively, according to the cardinalities of the given sets.

Applying the fuzzy quantification to mood AIA1 and the given example, potentially we can obtain 3 modified moods such as AII1, III1, IIA1. AII1 is valid mood for figure 1 . Thus potentially for the given mood we can increase $\tau$ from 0.4 to 1 , which is the actual objective of our fuzzification approach.

\subsection{Fuzzy-Syllogistic System (FSS)}

Based on the ideas discussed above, we have designed the software system FSS. The working cycle of the system is specified as follows:

Inductively accumulate sample instances of relationships between the objects M, P, S and classify them into the 96 distinct sub-sets.

Calculate the truth values of all 256 moods for these M, P, S relationships.

Apply fuzzy-quantification to all moods.

Use approximate reasoning (fuzzy sets), if results from previous step do not meet given requirements.

Currently, the system can load experimental input data from specific XML files. Thus, FSR can reason with any ontology that is in XML format, like OWL or RDF(S). 


\section{$4 \quad$ Ontologies}

The most popular definition of the concept ontology in information technology and AI community from a theoretical point of view is "A formal explicit specification of a shared conceptualization" or "An abstract view of the world we are modelling, describing the concepts and their relationships" [3].

Formally, an ontology may be defined as $\mathbf{O}=(\mathbf{C}, \mathbf{R}, \mathbf{A}, \mathbf{T o p})$, in which $\mathbf{C}$ is the non-empty set of concepts (including relation concepts and the $\mathbf{T o p}$ ), $\mathbf{R}$ is the set of all assertions, in which two or more concepts are related to each other, $\mathbf{A}$ is the set of axioms and Top is the most top level concept in the hierarchy. $\mathbf{R}$ itself is partitioned to two subsets, $\mathbf{H}$ and $\mathbf{N}$. $\mathbf{H}$ is the set of all assertions in which the relation is a taxonomic relation and $\mathbf{N}$ is the set of all assertions, in which the relation is a nontaxonomic relation[13].

Ontology construction is still an active topic of research. The major problems in building and using ontologies are the knowledge acquisition and the time-consuming construction [9].

\section{$5 \quad$ Ontology-Based Fuzzy-Syllogistic Reasoning}

Our objective is to implement syllogistic reasoning with ontologies and iteratively quantify ontological relationships with FSR. In this process, FSR does not directly produce an ontology. Concepts and relationships of a given ontology are evaluated and altered by the FSR.

Among the various possible ways to construct ontologies for a given domain, the most widely used approaches is the generation of an ontology from text-based sources [12]. There are several open-source tools for ontology generation from text corpora available for research purposes, such as Text2Onto [2], WebKB or DLLearner.

The most convenient tool for our purposes was Text2Onto, because it allows generating ontologies automatically and the generated ontology is sufficiently good.

\section{$5.1 \quad$ Building a Source Ontology}

For generating a source ontology it is necessary to prepare text corpus for the given domain. In case of Text2Onto, the text corpus may be a set of plain text documents, html pages and other unstructured or semi-structured text sources. The integration of this tool with the web search engine seems to be an optimal solution for collecting and preparing a text corpus for a given domain. Furthermore, as a result of stepwise synthesising concepts and properties, we obtain a domain ontology for the given corpus. The resulting ontology includes a set of nodes, which can represent terminal nodes or intermediate nodes, with linked relationships (Fig. 3 a).

\subsection{Building Graph of Dependencies}

For an existing ontology we can build a graph of syllogistic dependencies, which reflects quantitative relationships between concepts of the original ontology, such that FSR can be applied. 
Such a graph of dependencies contains all concepts and concept attributes of the original ontology and additionally all quantities that had contributed to their conceptualisations during the ontology learning process. The result is a quantified ontology.

Every attribute of a concept is further decomposed, e.g. new sub-concepts (subclasses) are created for each attribute value. This helps revealing hidden dependencies. All subclasses constructed from attributes must be linked with their parent class via a direct link (from attribute-class to parent).

Now we can link all concepts (classes) in our graph according to the bellow procedure. Let the number of all (sub)classes (terminal and intermediate) be equal to $\boldsymbol{N}$. For every subclass $\mathbf{S u b C l i}$ we need to consider $\mathbf{N}-\mathbf{1}$ subclasses. Analysis should be performed on pairs. For each pair of subclasses there are following possible conditions:

$\mathbf{S u b C l i}$ is subclass for $\mathbf{S u b C l j}$ (or otherwise) (Fig. 3 a, Subclass 2 and Subclass3): direct link from subclass to superclass on the graph of dependencies;

SubCli and SubClj have no shared subclasses (Fig. 3 a, Subclass1 and Subclass3): no link between the classes on the graph of dependencies;

SubCli and SubClj have shared subclasses (Fig. 3 a, Subclass3 and SubclassK): in this case we need to calculate fraction $\boldsymbol{F}$ of shared subclasses to number of subclasses for each of 2 subclasses (nodes), if $\boldsymbol{F}=\mathbf{1}$ for one of the nodes, then this node becomes subclass of the other node and we need to create a directed link from subclass to superclass; if $\boldsymbol{F}<\boldsymbol{1}$, we need to create a non-directed link (nodes have shared subclasses but no super classes of each other).

After performing these operations we will get the graph of dependencies, which is a reflection of the input ontology (Fig. 3 c).

There are four possible types of relationships between the classes (Fig. 3 b, 1-4 respectively):

directed link from CLASS_1 to CLASS_2: CLASS_2 includes all elements from CLASS_1, corresponds with A quantifier;

directed link from CLASS_2 to CLASS_1: CLASS_1 includes all elements from CLASS_2, corresponds with I quantifier (some elements of CLASS_1 in CLASS_2);

non-directed link from CLASS_1 to CLASS_2 (or otherwise): some elements from CLASS_1 in CLASS_2, at the same time, some elements from CLASS_2 in CLASS_1: corresponds with I, O quantifiers (appropriate quantifier can be selected according to cardinality of given sets);

no link between classes: corresponds with E quantifier.

FSR with such a dependency graph enables reasoning with 256 possible fuzzy inferences per triple concept relationships.

In some cases, the transitive concept of a triple can be removed, as that is not included in the conclusion. This helps reducing the complexity of the ontology and increases the level of abstraction over details that are no more required in reasoning. 

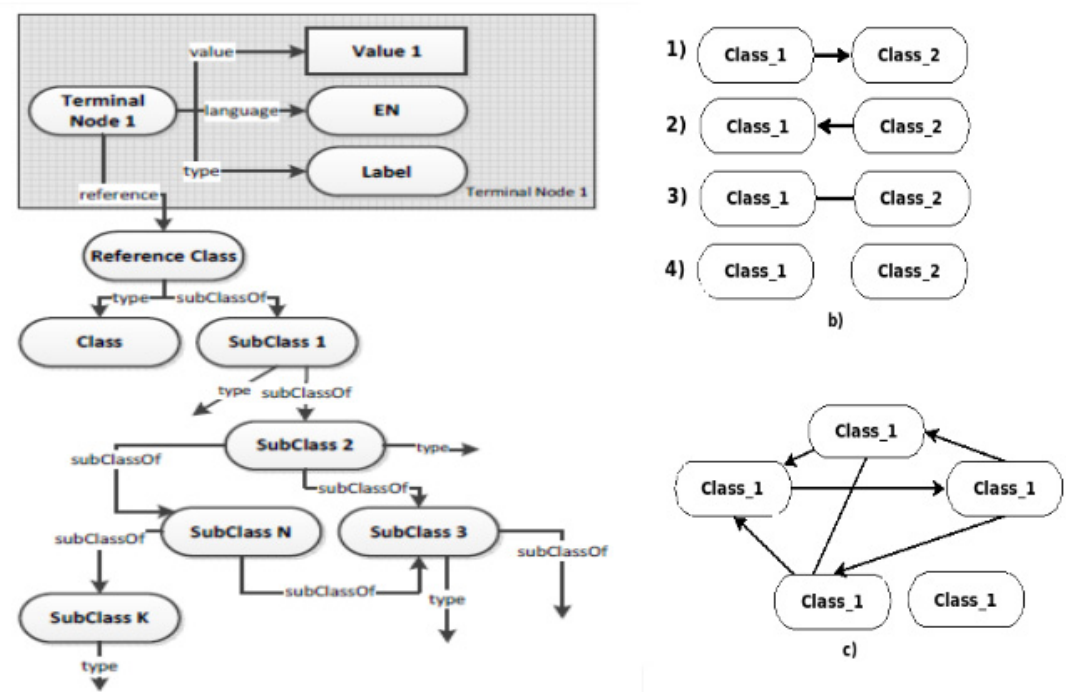

Fig. 3. Structural schema of a simple ontology (a); $1-4$ types of possible class relationships (b) and a sample graph of dependencies (c)

\subsection{Reasoning with Ontologies: Procedure}

Our objective is to prove the feasibility of FSR for reasoning with ontologies. The procedure consists of a few steps, such as rebuilding a given ontology in a format, such that always triple concepts are selected for analyses by the FSR component:

1. Calculate truth ratios of all 256 moods.

2. For given ontology, build dependency graph as described bellow.

3. Select triple of sets for analysis and label them with $\mathrm{M}, \mathrm{P}$ and $\mathrm{S}$.

4. Construct four syllogistic figures and associate quantifiers approriate for the quantities of the premises from the graph of dependencies; apply all possible quantifiers on conclusions.

5. Calculate truth ratios for all possible moods.

6. Select the moods with the highest truth ratio $\tau$; if $\tau<1.0$, try to apply fuzzification.

\subsection{Sample Application}

Let us perform the steps of the algorithm on a sample ontology (Fig. 4 a). One can see that there are four classes, Humans (with attribute \#gender), Philosophers, Scientists and Artists. Also there are six instances of the class Humans.

First of all, we need to distinguish all attributes of each class as separate subclasses. As shown on (Fig. 4 b), we have created 2 subclasses of Humans, humans_gender_male and humans_gender_female. Subclasses have direct link to their superclass, because these operation can be considered as decomposition of superclass, so all subclasses are part of superclass and relation between subclass and superclass. In terms of syllogistic quantification this is a A: all relationship. 


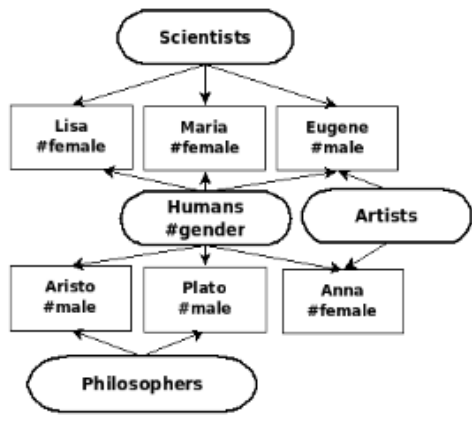

a)

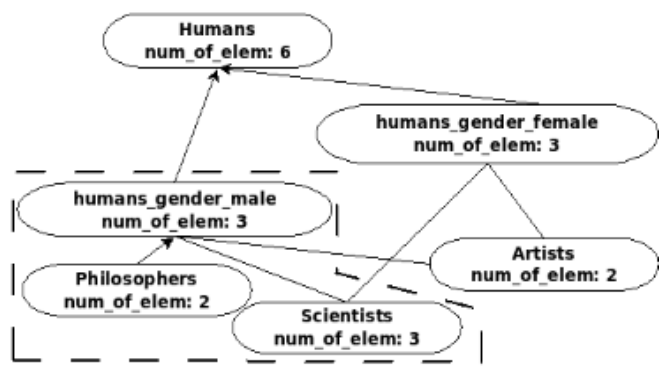

b)

Fig. 4. Sample ontology (a) and graph of dependencies for sample ontology (b)

After this we removed all instances of classes and calculate the cardinality (number of instances) for each (sub)class.

According to the algorithm above, we constructed direct links between classes. Now we can see that all PHILOSOPHERS are male (direct link from subclass to superclass), some of SCIENTISTS and ARTISTS are women, some are man. The constructed graph is suitable for performing FSR.

Now we need to select 3 classes. For now it looks quite indefinably, but when we embed our system in a real application, like an intelligent agent, the selected classes will be determined by the logic of the agent.

Lets consider the relationships between SCIENTISTS and PHILOSOPHERS classes through people_gender_male class (Fig. 4 b, dashed area).

According to the structure of syllogisms, the middle term (M) is humans_gender_male class, predicate $(\mathrm{P})$ is SCIENTISTS class and subject $(\mathrm{S})$ is PHILOSOPHERS class.

So, for four syllogistic figures we have following combinations:

\section{Figure 1}

$\{\mathrm{I}, \mathrm{O}\}: \mathrm{M} \mathrm{P}$

$\{\mathrm{A}\}: \mathrm{S} M$

$\{?\}:$ S P
Figure 2

$\{\mathrm{I}, \mathrm{O}\}: \mathrm{P} \mathrm{M}$

$\{\mathrm{A}\}: \mathrm{S} \mathrm{M}$

$\{?\}: S \mathrm{P}$
Figure 3

$\{\mathrm{I}, \mathrm{O}\}: \mathrm{M}$

$\{\mathrm{I}, \mathrm{O}\}: \mathrm{M} \mathrm{S}$

$\{?\}:$ S P
Figure 4

$\{\mathrm{I}, \mathrm{O}\}: \mathrm{P} M$

$\{\mathrm{I}, \mathrm{O}\}: \mathrm{M} \mathrm{S}$

\{?\}: S P

The problem is to find the most appropriate quantifier as conclusion. The quantifiers for the premises were selected according to the relationships between classes on the graph of dependencies. For example, direct link from Philosophers to humans_gender_male ( $\mathrm{S} M$ ) corresponds to the A quantifier, non-directed link from Scientists to humans_gender_male (P M) can be an I or O quantified relationship.

By calculating then truth ratios of all possible moods, we obtain the following results: 


\begin{tabular}{|c|c|c|c|c|c|}
\hline Figure 1 & \multicolumn{2}{|c|}{ Figure 2} & \multicolumn{2}{|c|}{ Figure 3} & Figure 4 \\
\hline $\mathrm{IAA}=0.285$ & IAA $=0.285$ & OIA $=0.084$ & IIA $=0.142$ & OIA $=0.134$ & IIA $=0.142$ \\
\hline IAE $=0.285$ & IAE $=0.285$ & $\mathrm{OIE}=0.157$ & $\mathrm{IIE}=0.144$ & $\mathrm{OIE}=0.104$ & $\mathrm{IIE}=0.144$ \\
\hline IAI $=0.714$ & IAI $=0.714$ & $\mathrm{OII}=0.845$ & $\mathrm{III}=0.885$ & $\mathrm{OII}=0.895$ & $\mathrm{III}=0.885$ \\
\hline IAA $=0.714$ & IAA $=0.714$ & $\mathrm{OIO}=0.915$ & $\mathrm{IIO}=0.857$ & $\mathrm{OIO}=0.865$ & $\mathrm{IIO}=0.857$ \\
\hline $\mathrm{OAA}=0.214$ & OAA $=0.333$ & $\mathrm{OOA}=0.128$ & $\mathrm{IOA}=0.183$ & $\mathrm{OOA}=0.194$ & IOA $=0.183$ \\
\hline $\mathrm{OAE}=0.357$ & $\mathrm{OAE}=0.333$ & $\mathrm{OOE}=0.185$ & $\mathrm{IOE}=0.154$ & $\mathrm{OOE}=0.134$ & $\mathrm{IOE}=0.154$ \\
\hline $\mathrm{OAI}=0.642$ & $\mathrm{OAI}=0.666$ & $\mathrm{OOI}=0.814$ & $\mathrm{IOI}=0.845$ & $\mathrm{OOI}=0.865$ & $\mathrm{IOI}=0.845$ \\
\hline $\mathrm{OAA}=0.785$ & $\mathrm{OAA}=0.666$ & $\mathrm{OOE}=0.871$ & $\mathrm{IOE}=0.816$ & $\mathrm{OOE}=0.805$ & $\mathrm{IOE}=0.816$ \\
\hline
\end{tabular}

The highest truth ratio is $\mathrm{OIO}=0.915$. We cannot apply fuzzy-quantification to a given mood, because it does not contain A or E quantifiers. Based on the bellow results we can restore the most suitable syllogism for the given data:
O: Male Scientists
I: Male Philosophers
O: Philosophers Scientist

with truth ratio $\tau=0.915$. Considering the OIO3 mood, we see that it has 71 cases, only 6 cases are false.

In analogy with previous example, consider following scenario: $M=$ humans_gender_male class, $\mathrm{S}=$ Philosophers, $\mathrm{P}=\mathrm{Humans}$. So, we want to investigate the relationship between Philosophers and Humans.

Possible moods are listed below:

\section{Figure 1}

$\begin{array}{ll}\{\text { A }\}: M P & \{I, O\}: P M \\ \{A\}: S M & \{A\}: S M \\ \{?\}: S \text { P } & \{?\}: S \text { P }\end{array}$

Figure 3

$$
\begin{aligned}
& \{\mathrm{A}\}: \mathrm{M} \mathrm{P} \\
& \{\mathrm{I}, \mathrm{O}\}: \mathrm{M} \mathrm{S}
\end{aligned}
$$$$
\{?\}: \mathrm{S} P
$$

Figure 4

$\{\mathrm{I}, \mathrm{O}\}: \mathrm{P} M$

$\{\mathrm{I}, \mathrm{O}\}: \mathrm{M} \mathrm{S}$

$\{?\}:$ S P

After calculating the truth ration for all moods, only three moods AAA1, AAI1 and AII3 have $\tau=1$ :
A: Male Humans A: Philosophers Male
$\{$ A, I $\}$ : Philosophers Humans
A: Male Humans I: Male Philosophers
I: Philosophers Humans

Actually, we can remove the link between PHILOSOPHERS and humans_gender_ men and create directed (or non-directed) link between PHILOSOPHERS and HUMANS. Performing the same operation for SCIENTISTS and ARTISTS classes, it is possible to remove all links to humans_gender_men. Since there is no link, related with the class, we can simply delete this class from the graph.

\section{$6 \quad$ Related Works}

Approximate reasoning [15] with ontologies is becoming increasingly popular in semantic web applications [9]. However, rather modus ponens or tollens-based fuzzifications are employed than categorical syllogisms.

Work on intermediate quantifiers [8], [10] is mostly theoretical, applications with ontologies are not known. 
The objective of probabilist ontologies is concerned with quality ontology generation [4], hence with improving reasoning as well, but these approaches too, do usually not involve categorical syllogisms.

\section{Conclusions}

We have discussed the fuzzy-syllogistic system (FSS) and introduced an application of fuzzy-syllogistic reasoning (FSR) with ontologies, which can be interpreted as a complex approximate reasoning approach that consists of 256 fuzzy inferences.

One future work is to expand the current restriction of two premises to $\mathrm{n}$ premises. FSR could then be applied transitively to all classes of the ontology, without decomposing the ontology into multiple triple relationships.

\section{References}

[1] Brennan, J.G.: A Handbook of Logic. Brennan Press (2007)

[2] Cimiano, P., Völker, J.: Text2Onto. In: Montoyo, A., Muńoz, R., Métais, E. (eds.) NLDB 2005. LNCS, vol. 3513, pp. 227-238. Springer, Heidelberg (2005)

[3] Gruber, T.R.: A translation approach to portable ontologies. Knowledge Acquisition 5(2) (1993)

[4] Ji, Q., Gao, Z., Huang, Z.: Reasoning with noisy semantic data. In: Antoniou, G., Grobelnik, M., Simperl, E., Parsia, B., Plexousakis, D., De Leenheer, P., Pan, J. (eds.) ESWC 2011, Part II. LNCS, vol. 6644, pp. 497-502. Springer, Heidelberg (2011)

[5] Kumova, B.İ., Çakır, H.: Algorithmic Decision of Syllogisms. Conference on Industrial, Engineering \& Other Applications of Applied Intelligent Systems (IEA-AIE 2010). Springer LNAI (2010)

[6] Kumova, Bİ., Çakir, H.: The fuzzy syllogistic system. In: Sidorov, G., Hernández Aguirre, A., Reyes García, C.A. (eds.) MICAI 2010, Part II. LNCS, vol. 6438, pp. 418 427. Springer, Heidelberg (2010)

[7] Kumova, B.I.: Symmetric Properties of the Syllogistic System Inherited from the Square of Opposition. Logica Universalis (2015)

[8] Murinová, P., Novák, V.: A formal theory of generalized intermediate syllogisms. Fuzzy Sets and Systems 186 (2012)

[9] Pan, J.Z., Thomas, E.: Approximating OWL-DL Ontologies. American Association for Artificial Intelligence (2009)

[10] Peterson, P.: On the logic of 'few', 'many', and 'most'. Notre Dame Journal of Formal Logic 20 (1979)

[11] Robinson, A., Voronkov, A.: Handbook of Automated Reasoning. Elsevier (2001)

[12] Shamsfard, M., Barforoush, A.A.: The State of the Art in Ontology Learning: A Framework for Comparison. The Knowledge Engineering Review 18(4) (2003)

[13] Sowa, J.F.: Knowledge Representation: Logical, Philosophical and Computational Foundations. Brooks/Cole (2000)

[14] Wille, R.: Contextual Logic and Aristotle's Syllogistic. Springer (2005)

[15] Zadeh, L.A.: Fuzzy Logic and Approximate Reasoning. Syntheses 30 (1975)

[16] Zadeh, L.A.: Syllogistic reasoning in fuzzy logic and its application to usuality and reasoning with dispositions. IEEE Transactions on Systems, Man and Cybernetics 15(6) (1985) 